This item was submitted to Loughborough's Research Repository by the author.

Items in Figshare are protected by copyright, with all rights reserved, unless otherwise indicated.

\title{
Evaluation of the quasi correlated tight-binding (QCTB) model for describing polyradical character in polycyclic hydrocarbons
}

PLEASE CITE THE PUBLISHED VERSION

https://doi.org/10.1063/1.4975196

PUBLISHER

AIP

VERSION

VoR (Version of Record)

\section{PUBLISHER STATEMENT}

This work is made available according to the conditions of the Creative Commons Attribution-NonCommercialNoDerivatives 4.0 International (CC BY-NC-ND 4.0) licence. Full details of this licence are available at: https://creativecommons.org/licenses/by-nc-nd/4.0/

\section{LICENCE}

CC BY-NC-ND 4.0

\section{REPOSITORY RECORD}

Luzanov, Anatoliy V., Felix Plasser, Anita Das, and Hans Lischka. 2019. "Evaluation of the Quasi Correlated Tight-binding (QCTB) Model for Describing Polyradical Character in Polycyclic Hydrocarbons". figshare. https://hdl.handle.net/2134/32125. 


\section{Evaluation of the quasi correlated tight-binding (QCTB) model for describing polyradical character in polycyclic hydrocarbons}

Anatoliy V. Luzanov, Felix Plasser, Anita Das, and Hans Lischka

Citation: The Journal of Chemical Physics 146, 064106 (2017); doi: 10.1063/1.4975196

View online: https://doi.org/10.1063/1.4975196

View Table of Contents: http://aip.scitation.org/toc/jcp/146/6

Published by the American Institute of Physics

\section{Articles you may be interested in}

Electron correlation by polarization of interacting densities

The Journal of Chemical Physics 146, 064113 (2017); 10.1063/1.4975329

Full-dimensional ground- and excited-state potential energy surfaces and state couplings for photodissociation of thioanisole

The Journal of Chemical Physics 146, 064301 (2017); 10.1063/1.4975121

Nonequilibrium diagrammatic technique for Hubbard Green functions

The Journal of Chemical Physics 146, 092301 (2017); 10.1063/1.4965825

Improved virtual orbitals in state specific multireference perturbation theory for prototypes of quasidegenerate electronic structure

The Journal of Chemical Physics 146, 064111 (2017); 10.1063/1.4975322

Generalized spin-ratio scaled MP2 method for accurate prediction of intermolecular interactions for neutral and ionic species

The Journal of Chemical Physics 146, 064108 (2017); 10.1063/1.4975326

Practical approximation of the non-adiabatic coupling terms for same-symmetry interstate crossings by using adiabatic potential energies only

The Journal of Chemical Physics 146, 064107 (2017); 10.1063/1.4975323

\section{AIP | The Journal of \\ AIP $\left.\right|_{\text {Chemical Physics }}$}

PERSPECTIVES 


\title{
Evaluation of the quasi correlated tight-binding (QCTB) model for describing polyradical character in polycyclic hydrocarbons
}

\author{
Anatoliy V. Luzanov, ${ }^{1, a)}$ Felix Plasser, ${ }^{2}$ Anita Das, ${ }^{3,4}$ and Hans Lischka ${ }^{3,4, b)}$ \\ ${ }^{1}$ SSI “Institute for Single Crystals," National Academy of Sciences of Ukraine, 60 Nauky Ave., \\ 61001 Kharkiv, Ukraine \\ ${ }^{2}$ Institute for Theoretical Chemistry, University of Vienna, Währingerstrasse 17, A-1090 Vienna, Austria \\ ${ }^{3}$ Department of Chemistry and Biochemistry, Texas Tech University, Memorial Circle \& Boston, Lubbock, \\ Texas 79409, USA \\ ${ }^{4}$ School of Pharmaceutical Sciences and Technology, Tianjin University, 92 Weijin Road, Nankai District, \\ Tianjin, 300072 People's Republic of China
}

(Received 28 October 2016; accepted 19 January 2017; published online 9 February 2017)

\begin{abstract}
We present a verification and significant algorithmic improvement of the quasi-correlation tightbinding (QCTB) scheme (a Hückel-Hubbard-type model mimicking electron correlation) for describing effectively unpaired electrons in the spirit of Head-Gordon's approach [M. Head-Gordon, Chem. Phys. Lett. 380, 488 (2003)]. For comparison purposes, results based on the high-level ab initio multireference averaged quadratic coupled cluster method previously computed in our works are invoked. In doing so, typical polyaromatic hydrocarbons (polyacenes, periacenes, zethrenes, and the Clar goblet) are studied. The evaluation shows that the QCTB Hückel-like scheme extended for electron correlation effects provides a qualitatively and in several cases also quantitatively good picture of the unpairing electrons in formally closed-shell electronic systems. Additionally, fairly large nanographene systems of triangulene structure $\left(\mathrm{C}_{426}\right)$ and a perforated nanoribbon $\left(\mathrm{C}_{8860}\right)$ have been treated at QCTB level. Two analytical model problems in the framework of QCTB prove the ability of this approximation to give a correct description of natural orbital occupancy spectra. For the studied QCTB scheme, an efficient algorithm is elaborated, and large-scale calculations of radical characteristics for nanographene networks with thousands of carbon atoms are possible. Published by AIP Publishing. [http://dx.doi.org/10.1063/1.4975196]
\end{abstract}

\section{INTRODUCTION}

Biradical states in organic chemistry have a long history, dating back to Tschitschibabin (Chichibabin) and Schlenk in early years of the 19th century. Now, biradicals (diradicals) and polyradicals have become an important subject of study in various fields, and the material science is a prominent one. Not only remarkable experimental research has been done (e.g., synthesis of high spin organic molecules ${ }^{1}$ ) but also modern quantum chemistry was enabled to keep pace with the rapid advance of organic radical chemistry, and a plethora of original quantum chemistry models and ideas on the biradical/polyradical theory was produced such as spin-flip methods, ${ }^{2,3}$ projected Hartree-Fock theory, ${ }^{4}$ active-space variational two-electron reduced-density-matrix (2-RDM), ${ }^{5,6}$ density matrix renormalization group (DMRG), ${ }^{7}$ multireference averaged quadratic coupled cluster (MR-AQCC) approach, ${ }^{8-10}$ and coupled cluster with singles, doubles, and noniterative triples $\operatorname{CCSD}(\mathrm{T}){ }^{11}$

In current terminology, radical species are the case of electronic open-shells. An interesting special issue of this kind is singlet open-shell systems. The treatment of the latter turned out to be a not so simple matter because singlet ground states are mainly closed-shells in its nature, and only sufficiently strong electron correlations can cause unpairing

\footnotetext{
a)E-mail: luzanov@xray.isc.kharkov.com

b) E-mail: hans.lischka@univie.ac.at
}

in electron shells, creating open-shell electronic singlet structures. Happily, the current quantum chemistry approaches can deal with large molecular systems and can, to a large extent, also take electron correlation explicitly into account (see previous paragraph and Refs. 12-14). Nevertheless, material science problems, in particular for molecular nanosized materials, demand treating polyatomic clusters with hundreds and more atoms. The typical example of such materials is nanographenes. ${ }^{15}$ These kinds of systems are still out of reach or at least extremely challenging for serious ab initio calculations.

Diagnostics of radicaloid states in formally closed-shell molecules are an intriguing theme, which deserves further study. One of the main directions here is the effectively unpaired electron (EUE) theory originated in papers. ${ }^{16-21}$ The main quantity of the EUE theory is the number of unpaired electrons and respective EUE distribution function over the molecule. Many interesting EUE applications appeared in the literature and most of them are devoted to the EUE diagnostics in polycyclic aromatic hydrocarbons (PAHs) and similar graphene-like molecules ${ }^{7,12-14,22,23}$ (see also a recent review ${ }^{24}$ ). It should be stressed that only high-level ab initio EUE theory can provide a most reliable basis for the entire EUE concept. ${ }^{12,13}$ Nevertheless, semiempirical models, even very simplistic, such as one proposed in Refs. 24 and 25 can give a seemingly reasonable description of the unpairing phenomenon. 
Evidently, when treating very large-scale systems, semiempirical schemes have an advantage over the extremely expensive $a b$ initio approaches. But before using a certain semiempirical scheme, a careful analysis of its weak and strong sides is necessary. In Refs. 24 and 25 , we used a quasi-correlation tight-binding (QCTB) model of Hückel-like type, which was previously developed for $\pi$-electron diamagnetism. ${ }^{26}$ Some applications of EUE to graphene molecules and a very restricted comparison with ab initio results from Ref. 12 gave only a preliminary assessment of QCTB as a seemingly satisfactory approach to the EUE problems. ${ }^{24}$

This consideration has motivated us to carry out an extended comparative analysis of the ab initio and QCTB levels of EUE theory and to present also significant improvements in the efficiency of the QCTB algorithm which is especially important for the computation on very large molecular systems. As to the ab initio approach, a quite reliable approach turned out to be the already mentioned MR-AQCC scheme which allows the balanced inclusion of quasi-degenerate configurations in the reference wave function and of dynamic electron correlation including size-extensivity contributions. This approach was intensively used in a series of papers ${ }^{12,14,27-29}$ on the description of EUE in large graphene-like and related PAH molecules. In the present paper, AQCC will serve as a reference scheme with which the approximate semiempirical approaches may be compared and assessed.

The plan of the paper is as follows. In Sec. II, the key notions of the unpaired electron theory are briefly considered. The elementary Hückel-like description of EUE effects (QCTB model) is given in Section III. The computational details are presented in Section IV, and the comparable analysis of polyacenes, phenacenes and graphene-like molecules is furnished in Sections V and VI. In Section VII, we discuss EUE effects in giant nanographene structures that demonstrate possibilities of QCTB to treat the systems which are simply unfeasible for rigorous ab initio methods. In Sec. VIII we sum up the results and give an outlook for further development. Furthermore, in Appendix A two simple analytical models are examined. They clarify conceptually the interplay between $\pi$-orbital energy gap and related features of natural orbital occupation spectra. Finally, in Appendixes B and C, we propose a more efficient algorithm than previously given for QCTB in Refs. 24 and 25.

\section{FORMAL PRELIMINARIES}

In this section, without going into more details, we sketch the main EUE notions and related issues important for practice. We follow the basic papers ${ }^{16-21}$ and, in part, review. ${ }^{24}$ Below, only one-electron notions will be considered for the given spinsinglet $N$-electron molecule. The key quantities here are total number, $N_{\mathrm{U}}$, of the unpaired electrons, and respective unpaired electron density matrix $D_{\mathrm{U}}$ from which an atomic distribution of unpaired electrons over molecule can be derived.

Generally, $N_{\mathrm{U}}$ for singlet states can be defined as a numerical measure of deviation of the given density matrix $D$ from being idempotent in a spin-orbital frame, or being duodempotent in a spin-free one. The first measure of this kind is the Yamaguchi index ${ }^{16}$ which simply quantifies the duodempotency condition

$$
2 D-D^{2}=0,
$$

by the matrix trace of the form $\operatorname{Tr}\left(2 D-D^{2}\right)$. A finer $N_{\mathrm{U}}$ index is due to Head-Gordon. ${ }^{20,21}$ The most popular version of this index, which we denote by $N_{\mathrm{U}}^{\mathrm{lin}}$, is defined as follows:

$$
N_{\mathrm{U}}^{\operatorname{lin}}=\sum_{j} \operatorname{Min}\left[n_{j}, 2-n_{j}\right]
$$

where $n_{j}$ are the natural orbital occupation numbers (NOONs), that is, eigenvalues of $D$.

Independently, a hole-particle EUE index, $N_{\mathrm{h}-\mathrm{p}}$, was introduced, ${ }^{30,31}$ based on one interesting paper of Kutzelnigg and Smith. ${ }^{32}$ It turned out that for ground singlet states (our main concern here), the identity $N_{\mathrm{U}}^{\mathrm{lin}}=N_{\mathrm{h}-\mathrm{p}}$ is valid, ${ }^{24,33}$ and we will not distinguish between these quantities, although they are not identical in the case of excited states. ${ }^{24}$ Thus, we can replace Eq. (1) by the equivalent spectral sum

$$
N_{\mathrm{U}}^{\mathrm{lin}}=2 \sum_{j>N / 2} n_{j}
$$

Here sum is over "virtual" natural orbitals, and $N / 2$ is the number of the electronic pair in the even-numbered $N$-electron molecule under study. Thus, for $j>N / 2$ numbers $n_{j}$ are the populations of virtual natural orbitals, and for $j \leq N / 2$ numbers $2-n_{j}$ are the populations of the corresponding hole orbitals. The above given hole-particle interpretation of the HeadGordon index clears up a somewhat formal definition (1).

Although $N_{\mathrm{U}}^{\text {lin }}$ is a good indicator of radical character for semiempirical models, ${ }^{24,25,33}$ the index overestimates this character in the case of correlated ab initio calculations. ${ }^{12-14,27-29}$ This problem derives from the fact that dynamic correlation leads to a large number of NOs with occupations close to, but not exactly equal to, 2 and 0 , which, in total, may have a stronger effect on Eq. (2) than the actual open-shell electrons. To overcome this problem, we use the modified EUE measure

$$
N_{\mathrm{U}} \equiv N_{\mathrm{U}}^{\mathrm{nonlin}}=\sum_{j}\left(2 n_{j}-n_{j}^{2}\right)^{2},
$$

also proposed by Head-Gordon ${ }^{21}$ as this formula effectively damps the NOONs close to 2 and 0 . These NOONs are insignificant for understanding the radical nature, and using Eq. (3) leads to a more consistent picture, as demonstrated in the above cited works based on ab initio computations. It is essential that Eq. (3) is but the squared norm of duodempotency condition: $N_{\mathrm{U}}=\left\|2 D-D^{2}\right\|^{2}$.

Together with this, our results ${ }^{24,25,33}$ within semiempirical $\pi$-electron models tell us that the primary, $N_{\mathrm{U}}^{\text {lin }}$, index is a quite appropriate EUE measure for $\pi$-electrons within the classical Pariser-Parr-Pople (PPP) approximation. In this case, the typical $\pi$-parametrization scheme with two-electron twocenter integrals due to Ohno turns out to be, so to say, the soft parametrization scheme which partly incorporates dynamic electron correlation effects into the properly chosen semiempirical parameters. Then, within the adopted semiempirical framework, the dynamical correlation effects are markedly suppressed from the very beginning, and $N_{\mathrm{U}}^{\text {lin }}$ becomes a suitable measure. We see that no universal recipe exists for choosing an optimal $N_{\mathrm{U}}$ variant (see also the discussion in Section 
6.14 of Ref. 24). Nevertheless, in the present paper to be more consistent and clear, we employ $N_{\mathrm{U}}$, Eq. (3), for both $a b$ initio and the semiempirical theories when making comparison between them.

\section{THE QUASI-CORRELATED TIGHT BINDING SCHEME FOR EUE}

Here we briefly outline main points of QCTB in the context of EUE properties. ${ }^{24,25}$ The precursor of QCTB is the Hubbard-like model of Langer and Mattis ${ }^{34}$ (see also a very similar consideration in Ref. 35). In a sense, their approach is a simplification of the usual alternant MO (AMO) theory where a spin-projection procedure is neglected (the spin projection is needed in the consistent AMO theory of Löwdin ${ }^{36}$ ). By construction, AMO theory is formulated for alternant systems, that is, for unsaturated hydrocarbons having no odd carbon cycles. In such molecules, the Hückel spectrum of orbital energies is symmetric in respect to a zero value (the energy of a lone $\pi$-orbital). This is the so-called Coulson-Rushbrooke parity theorem. ${ }^{37}$ Thus, it is sufficient to work with positive orbital energies $\varepsilon_{j}(j>N / 2)$ corresponding to vacant $\pi$-MOs. Throughout the paper, the Hückel spectrum is considered as being expressed in terms of the standard resonance integral of a $\mathrm{C}-\mathrm{C} \pi$-bond $\left(\beta_{0}\right)$.

In the AMO scheme of Langer and Mattis, the $\varepsilon_{j}$ are renormalized, $\varepsilon_{j} \rightarrow \tilde{\varepsilon}_{j}$, by the introduction of a certain splitting parameter $\delta$ producing an antiferromagnetic ordering. ${ }^{34}$ The renormalized $\tilde{\varepsilon}$ are of the form

$$
\tilde{\varepsilon}_{j}=\sqrt{\left(\varepsilon_{j}\right)^{2}+\delta^{2}} .
$$

For finding the splitting parameter, we must solve a nonlinear equation (a self-consistency requirement) which has nontrivial solutions only for sufficiently strong electron correlation (more exactly, when a triplet instability ${ }^{38,39}$ occurs). This peculiarity excludes from the consideration, the important class of weakly correlated systems. At the same time, the nontrivial solution for $\delta$ always exists in the full (with spin-projection) AMO method. A possible, while a crude way to obviate this difficulty is to fix $\delta$ as a fitting parameter of the theory. This is done in our approach ${ }^{25}$ and was termed the quasi-correlation tight-binding (QCTB) approach derived from somewhat different assumptions. ${ }^{26}$ In practice, we take the value $\delta=7 / 24$ which was chosen so as to reproduce the EUE characteristics in pentacene computed within the $\pi$-coupled cluster doubles (CCD) method.

Having at disposal renormalized orbital energies (4), that is, eigenvalues of a renormalized one-electron Hamiltonian, the corresponding density matrix is easy to find. ${ }^{24}$ The final result for the "vacant" part of the NOON spectrum is

$$
n_{j}^{\mathrm{QCTB}}=1-\varepsilon_{j} / \tilde{\varepsilon}_{j}, \quad j>N / 2, \varepsilon_{j} \geq 0,
$$

and the corresponding "occupied" part of the NOON spectrum is $2-n_{j}^{\mathrm{QCTB}}=1+\varepsilon_{j} / \tilde{\varepsilon}$. Respectively,

$$
N_{\mathrm{U}}^{\mathrm{QCTB}}[\operatorname{lin}]=2 \sum_{j>N / 2} n_{j}^{\mathrm{QCTB}},
$$

and the nonlinear EUE index (3) is of the form

$$
N_{\mathrm{U}}^{\mathrm{QCTB}}=\sum_{j}\left[2 n_{j}^{\mathrm{QCTB}}-\left(n_{j}^{\mathrm{QCTB}}\right)^{2}\right]^{2},
$$

This $N_{\mathrm{U}}^{\mathrm{QCTB}}$ will be our main semiempirical EUE quantity in the subsequent analysis.

The detailed comparison of QCTB with AQCC will be given in Secs. IV-VI. It is underlined that only the Hückel orbital spectrum is needed for calculating the above considered EUE indexes at QCTB level. We also notice principal features of Eq. (5) for NOONs. As well known, the Hückel spectrum $\left\{\varepsilon_{j}\right\}$ is localized in the interval $\left[-\varepsilon_{0}, \varepsilon_{0}\right]$ where for the planar $\pi$-systems, $\varepsilon_{0}=3$ (a maximum of neighboring atoms). At the same time, in large systems, HOMO and LUMO energies can be concentrated near zero. Putting in Eqs. (4) and (5) value $\varepsilon_{j}= \pm 3$, and $\delta=7 / 24$, we get maximal and minimal possible values of $n_{j}^{\mathrm{QCTB}}$ near 2 and zero (more exactly, 1.9953 and 0.0047). Clearly, the value $n_{j}^{\text {QCTB }}$ near 2 is typical for doubly occupied MOs in weakly correlated closed-shell systems. Another case is when $\varepsilon_{j}=0$, that occurs for nonbonding occupied MO. Then Eqs. (4) and (5) automatically lead to $n_{j}^{\mathrm{QCTB}}=1$. The latter exactly corresponds to the presence of an unpaired electron. We see that the given Hückel-like theory based on the QCTB formalism, which is very elementary in its nature, catches correctly the crucial EUE features of $\pi$ electron radicals or polyradicals. The concrete results given in Secs. IV-VII confirm this.

\section{COMPUTATIONAL DETAILS}

All ab initio reference data were computed previously and were performed at the multireference averaged quadratic coupled cluster (MR-AQCC) level of theory. ${ }^{89}$ For the polyacenes, a restricted active reference space composed of 16 active electrons in 16 active orbitals was found to be necessary in order to describe the open shell character of the polyacene series properly. ${ }^{12}$ Since a CAS $(16,16)$ reference would have been too costly, a RAS(6)/CAS(4,4)/AUX(6)-1ex scheme was used where RAS stands for restricted active space containing six orbitals which are initially doubly occupied, AUX for auxiliary space, and CAS for complete active space. The 16 active electrons were distributed over the remaining orbitals with the restrictions that at least 11 electrons were in RAS (single excitations), and at most one was located in AUX. For the periacene systems, ${ }^{12}$ a complete active reference space of 8 electrons in 8 orbitals was found to be adequate. Within the MR-AQCC calculations, all single and double excitations out of these reference spaces were allowed within the $\pi$-space whereas all $\sigma$ orbitals were frozen in these calculations. The 6-31G basis set was employed in the polyacene and periacene MR-AQCC calculations. The results for dibenzoheptazethrene obtained in Ref. 14, a CAS $(4,4)$ reference space and the larger 6-311G(2d) basis had been used. In this latter work, it had also been demonstrated that for phenalenyl, heptazethrene dibenzoheptazethrene singlet/triplet splittings, NOON and EUE values agreed closely between the $6-31 \mathrm{G}, 6-31 \mathrm{G}^{*}$, and $6-31 \mathrm{G}(2 \mathrm{~d})$ basis sets. Additionally, freezing of the $\sigma$ orbitals did not affect the results either. These computations were performed within the COLUMBUS program system. ${ }^{10}$ Wavefunction analysis 
was performed within COLUMBUS, and additionally, within the TheoDORE package. ${ }^{40-42}$

In the case of the semiempirical description, QCTB is our main $\pi$-electron scheme which can be applied only for alternant hydrocarbons (bipartite graphs in graph-theoretic terms). QCTB has the single fitting parameter $\delta$ which, in the computations, will be taken to be 7/24 (in units of the resonance integral $\beta_{0}$ ). The orbital energy spectrum $\left\{\varepsilon_{j}\right\}$ needed for QCTB Equations (5)-(7) is obtained by solving the standard eigenvalue problem for the Hückel Hamiltonian matrix. Up to a factor $\beta_{0}$, the latter is equivalent to an adjacency matrix of the respective molecular graph. Recall that nonzero nondiagonal elements of the adjacency matrix are assigned a unity value only if the corresponding two vertices of the graph ( $\pi$-centers) are neighboring. Many analytically solved cases of the graph spectra are covered in Ref. 43.

We will consider examples of different-type NOON spectra. The first is related to weakly correlated systems, when most occupied NOONs are near 2 and most virtual NOONs are near zero. The phenacenes studied in Ref. 12 are of this type. In the case of sufficiently strong $\pi$-electron correlations, a few occupied NOONs deviate markedly from a value of 2 , and this occurs in the acenes. A comparable analysis of semiempirical vs $a b$ initio EUE theories will be given in the rest of the paper.

One additional issue is pertinent to be cleared now. This concerns the question what indexes are preferable, the total values $N_{\mathrm{U}}$ or the specific values ( $N_{\mathrm{U}}$ per electron) which we denote by $\bar{N}_{\mathrm{U}}$. It seems that the reply depends on the systems we analyze. If the electrons are weakly correlated, $N_{\mathrm{U}}$ can be misleading. Really, even in very stable nonradical but large systems, inevitable small effects of dynamical electron correlation produce marked values of $N_{\mathrm{U}}$ (in both, linear and nonlinear, versions). Let us take as an example of the known PAH $\mathrm{C}_{78} \mathrm{H}_{26}$ which is the fully benzenoid hydrocarbon of the Clar type. ${ }^{44}$ We find that $N_{\mathrm{U}}^{\mathrm{QCTB}}=1.073$, but this value indicating a semiradical type has no relation to the above stable hydrocarbon. At the same time, a small value $\bar{N}_{\mathrm{U}}^{\mathrm{QCTB}}=0.014$ is more understandable, being almost the same as in the stable phenacenes (see Section V). Another case is the highly correlated systems where the large values of $N_{\mathrm{U}}$ indeed reflect their genuine high radical character. In this paper, we will discuss both indexes: $N_{\mathrm{U}}$ and $\bar{N}_{\mathrm{U}}$. Additional to $N_{\mathrm{U}}$ and NOON spectra, the EUE atomic distribution is very informative from the chemical point of view. The distribution is derived from the corresponding $D_{\mathrm{U}}$ matrix. In $\pi$-models it is computed in a usual way from the standard LCAO coefficients $C_{\mu j}$ (in the orthonormal basis of $\pi$-AOs)

$$
D_{\mu}^{\mathrm{U}}=\sum_{j}\left|C_{\mu j}\right|^{2}\left[2 n_{j}^{\mathrm{QCTB}}-\left(n_{j}^{\mathrm{QCTB}}\right)^{2}\right]^{2} .
$$

More efficient QCTB algorithms, which are proposed in Appendixes B and C, can be used if NOON spectra are not required and only $N_{\mathrm{U}}^{\mathrm{QCTB}}$ and EUE densities are needed. We used these algorithms for giant nanographenes in Section VII.

\section{ACENES AND PHENACENES}

Here we consider systematically EUE in the specific PAHs based on AQCC and QCTB computations. The linear [n] acenes are very popular systems, which have been most intensively studied within the EUE problem as well. $7,12,21,24,33$ At the QCTB level they are elementarily treated with help of the Hückel spectrum given analytically by Coulson ${ }^{45}$

$$
\varepsilon_{j}= \pm\left(1 \pm\left[9+8 \cos [\pi j /(n+1)]^{1 / 2}\right) / 2\right.
$$

where $j=1,2, \cdots, n$ ( $n$ is the number of benzenoid cycles), and there are also two additional solutions: $\varepsilon= \pm 1$. Besides, by using a suitable matrix formula for $N_{\mathrm{U}}$ in the form of Eq. (B1), the explicit representation by a rational function in $\delta$ is obtained for a few first members of the polyacene series (see Table S1 in the supplementary material). The key peculiarity of Eq. (9) is a vanishing orbital energy gap in asymptotically large [n] acenes $\left(\varepsilon_{n} \rightarrow 0\right.$ when $\left.n \rightarrow \infty\right)$. This is a qualitatively different type of behavior than that of the isoelectronic phenacenes which we also examine in this section.

The phenacenes are interesting in their own right. They are related to a particular case of PAHs with fairly small electron correlation effects relative to the isoelectronic acenes and are quite tractable within the one-electron approach. Simple structural reasonings make clear why such PAHs have a unique stability among other ones. For instance, the number of Clar sextets increases linearly with the phenacene chain length as opposed to the acenes (for applications of the Clar sextet theory to PAHs and nanographenes see also Refs. 46 and 47). In fact, the same conclusion follows from the counting number of Kekulé structures: the number exponentially increases with $n$ for [n]phenacenes, but linearly with $n$ in [n]acenes).$^{48}$ It is also important that in the phenacenes the singlet-triplet gap stays nearly constant ${ }^{49}$ with an increase of $n$ whereas in the acenes a very marked decrease is observed.

The $N_{\mathrm{U}}$ value for low-order linear acenes and phenacenes is plotted in Fig. 1 (and the data are also presented in Tables $\mathrm{S} 2$ and S3 of the supplementary material). Fig. 1 illustrates the striking difference in electronic structure properties between the two types of isomeric systems. Whereas, the AQCC $N_{\mathrm{U}}$ value stays below 0.5 in the case of the phenacenes, a strong linear increase is observed for the acenes. The QCTB method does not only reproduce the qualitative trends but is even in good quantitative agreement with the AQCC reference, disregarding the even stronger increase of the AQCC values

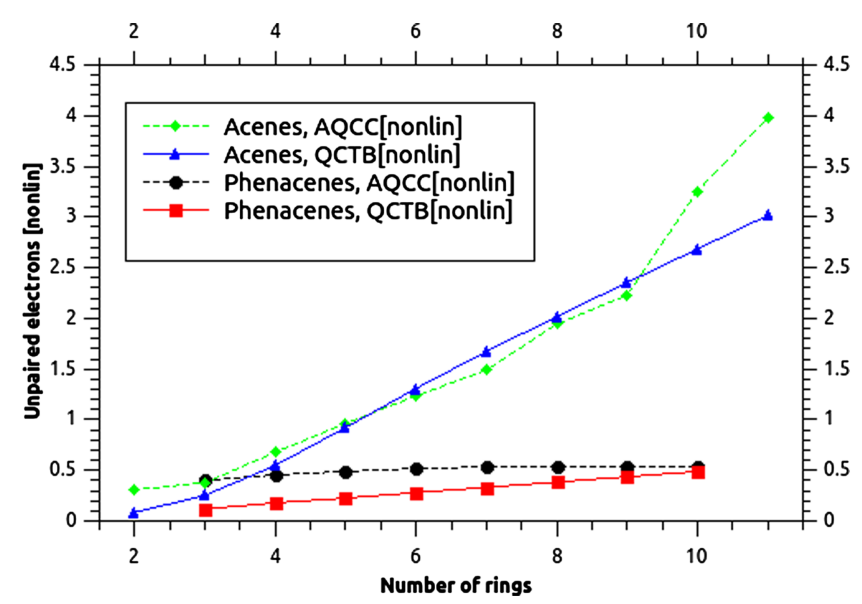

FIG. 1. Comparison of the size dependence of $N_{\mathrm{U}}^{\mathrm{AQCC}}$ and $N_{\mathrm{U}}^{\mathrm{QCTB}}$ for linear acenes and phenacenes. 
for $n=9$ and 10 which might be an artifact of a limited size of the reference space. Even more, the individual NO eigenvalue spectra show a close resemblance (Fig. S1 of the supplementary material). A notable difference is only observed in the case of the phenacenes (for NOON spectra, see Fig. S2 of the supplementary material). Here, QCTB starts at a rather low value of $N_{U}=0.12$ and shows a steady increase to 0.49 whereas AQCC varies in a narrower range (between 0.40 and 0.54). However, it should be pointed out that an accurate description of dynamic correlation is not the target of either of these methods.

Before concluding this section, we underline a principal feature of QCTB to provide a rather general EUE theory for gapless and gap-containing $\pi$-electron systems. The corresponding analytical consideration is given in Appendix A where explicit expressions for NOONs and EUE indexes are obtained for the two different, general-type models of $\pi$-electron orbital spectrum. These results well agree with the main characteristic traits of large polyacenes and polyphenacenes.

\section{PERIACENES AND INTRINSICALLY POLYRADICAL STRUCTURES}

In Figs. 2 and 3, and Table S4 of the supplementary material, we present for the $(3 \mathrm{a}, \mathrm{nz})$ and $(5 \mathrm{a}, \mathrm{nz})$ periacene series a comparison of $N_{\mathrm{U}}$ and NOON results between AQCC and QCTB calculations. Because these systems are of strong correlation type, we observe here a comparably good qualitative agreement between both approaches. The $N_{\mathrm{U}}$ values (Fig. 2) show a quasi-linear increase for both periacene systems with an enhanced increase given by the AQCC method as compared to QCTB. A similar evolution in polyradical character as measured by deviation of NOONs from zero and two, respectively, is observed in Fig. 3.

To be complete, we document in Fig. 4 the good agreement of the AQCC and QCTB EUE distributions for two representative examples, the [11] acene and the $(5 \mathrm{a}, 6 \mathrm{z})$ periacene.

Such distributions, to a large extent, depend on a contribution of HONO, that is, the high occupied natural orbital (ala HOMO), and LUNO, that is, the corresponding counterpart of LUMO. Within our $\pi$-scheme, we have HONO $=$ HOMO and LUNO = LUMO, so all NOs are the Hückel ones, whereas generally $\mathrm{HONO} \neq \mathrm{HOMO}$ and LUNO $\neq$ LUMO in $a b$ initio approaches. In Fig. 5 we compare HONO of the $(5 \mathrm{a}, 5 \mathrm{z})$ periacene treated by both our approximations. While the images are quite similar, we remark that in the QCTB case, the HONO is a bit more delocalized than in the AQCC case.

In our comparisons, we also address several systems which recently became attractive to experimentalists as well as to theoreticians. The zethrenes are of this type. ${ }^{14,50-53}$ We restrict ourselves to displaying densities of unpaired
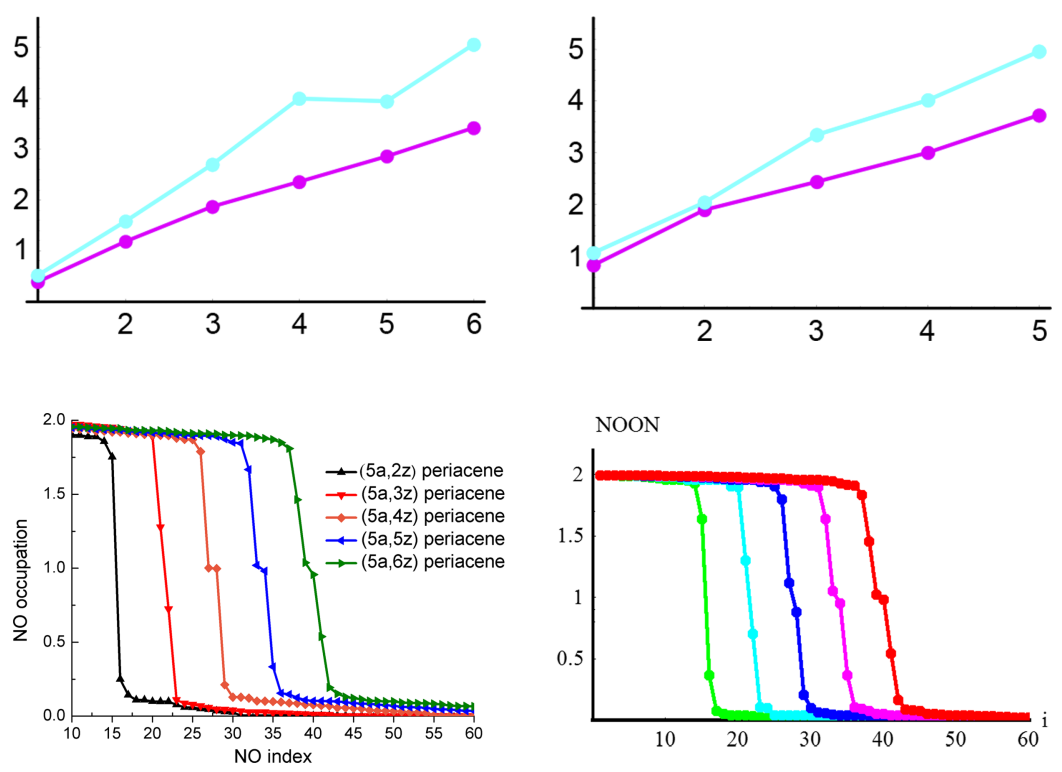

FIG. 2. Size dependence of $N_{\mathrm{U}}^{\mathrm{AQCC}}$ (cyan) and $N_{\mathrm{U}}^{\mathrm{QCTB}}$ (magenta) in (3a,nz) periacenes (left panel) and in $(5 \mathrm{a}, \mathrm{nz})$ periacenes (right panel).

FIG. 3. NOON spectra for $(5 \mathrm{a}, \mathrm{nz})$ periacenes at the AQCC level (left panel, reprinted with permission from Plasser et al., Angew. Chem., Int. Ed. 52, 2581 (2013). Copyright 2013 John Wiley and Sons) and the QCTB level (right panel). periacene:
AQCC

[11] acene :
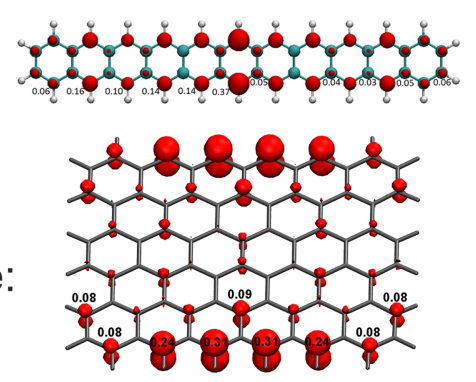

QCTB

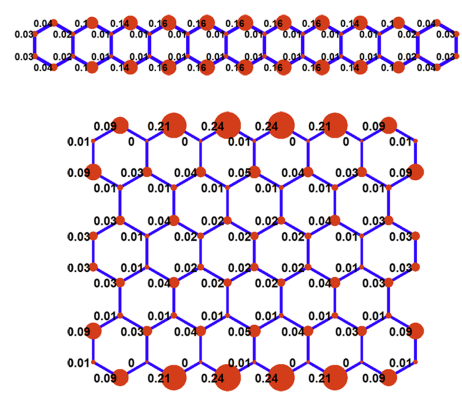

FIG. 4. Comparison of the unpaired electron density in the acene and periacene. AQCC periacene results: Reprinted with permission from Plasser et al., Angew. Chem., Int. Ed. 52, 2581 (2013). Copyright 2013 John Wiley and Sons. 
AQCC HONO

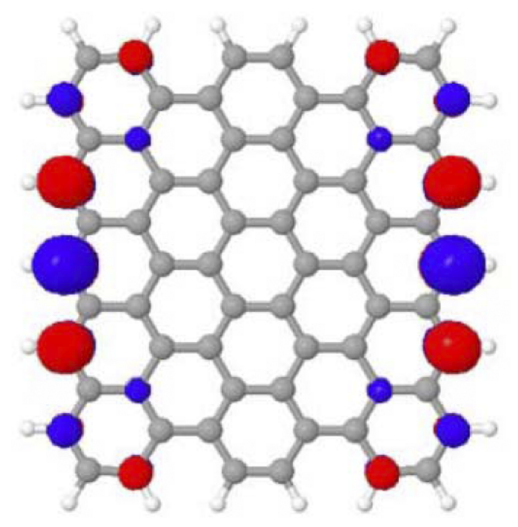

QCTB HONO

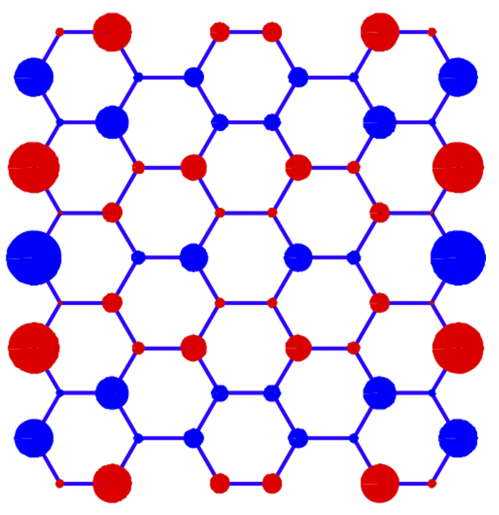

FIG. 5. Comparison of HONO in the $(5 \mathrm{a}, 5 \mathrm{z})$ periacene.

electrons for dibenzoheptazethrene (Fig. 6). Again, for this strongly correlated system, we observe good agreement between the two theories used in this work. Importantly, the most active centers of the EUE localization are the same in both approaches. At the same time, in the case of the heptazethrene (Fig. S3 in the supplementary material), the QCTB results are only qualitatively the same, and the atomic EUE densities are too large in comparison with the AQCC data.

Another proper biradical system is the well-known Clar goblet structure which we recently studied by AQCC method in Ref. 14 as well. In Fig. 7 we compare the AQCC densities of unpaired electrons with those obtained now in QCTB, and again we find a satisfactory picture being proposed by the semiempirical model.

\section{LARGE-SCALE SYSTEMS}

In addition to the discussion of polymers and related problems in Secs. IV-VI and in Appendix A, the large-scale systems which are not polymeric type deserve an additional study in the EUE context. Such studies might reveal possible types of behavior of unpaired electrons in complex networks, and equally they might show how QCTB is applicable over a wide size range of nanostructures. In this context, we mention large non-Kekulean structures, especially those of the triangulene-like series which have attracted the attention of the graphene community in connection with the design of possible graphene-based spintronic devices. ${ }^{54-58}$ In this field, a more expressive term "topological frustration"

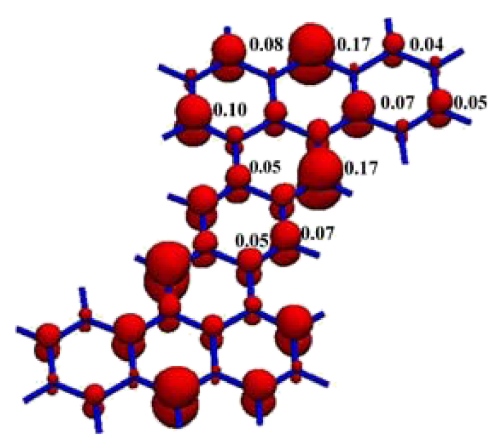

AQCC

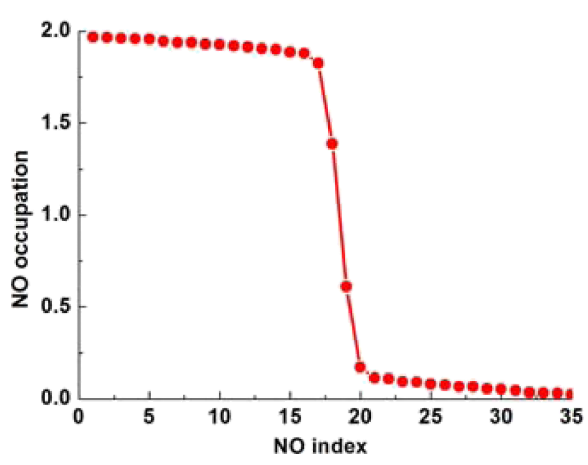

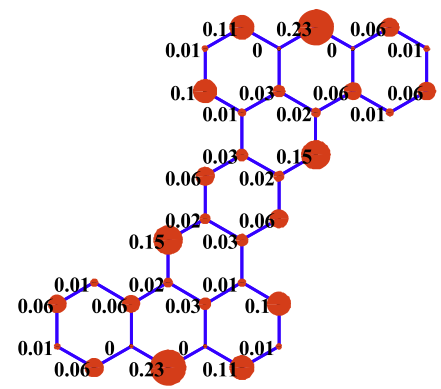

\section{QCTB}

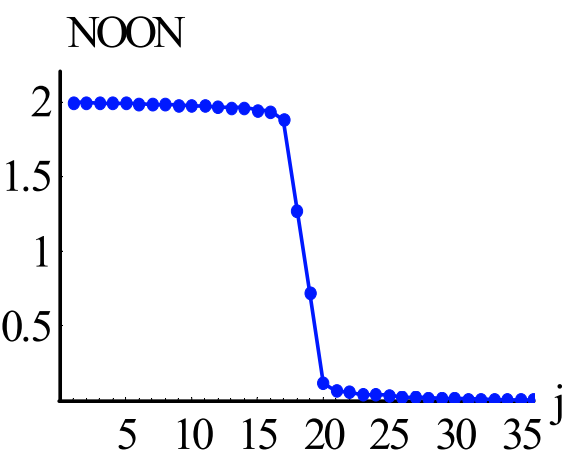

FIG. 6. Comparison of AQCC (left panels) $^{14}$ and QCTB (right panels) for densities of unpaired electrons and $\pi$-electron NOON spectrum in the 5,6:13,14-dibenzoheptazethrene; $N_{\mathrm{U}}^{\mathrm{AQCC}}=2.24, N_{\mathrm{U}}^{\mathrm{QCTB}}=1.93 . \mathrm{AQCC}$ densities of unpaired electrons results: Reprinted with permission from Das et al., J. Phys. Chem. A 120, 1625 (2016). Copyright 2016 American Chemical Society. 
AQCC

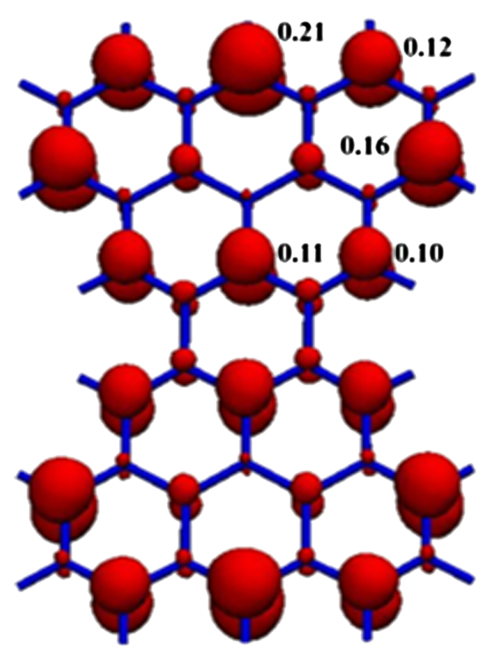

\section{QCTB}

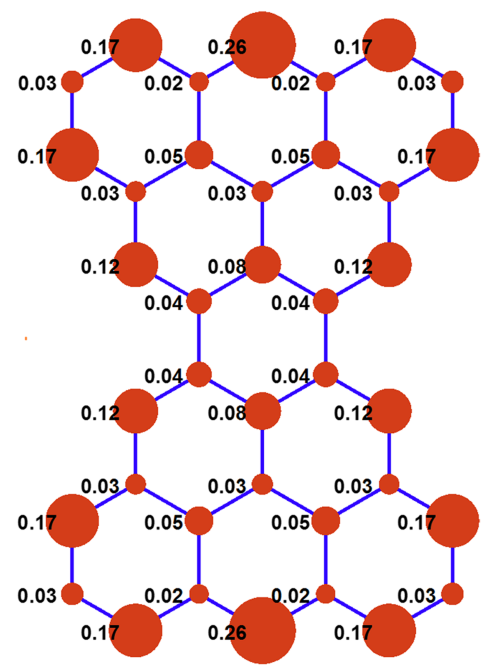

FIG. 7. Comparison of AQCC (left panel) and QCTB (right panel) densities of unpaired electrons in the Clar goblet. $N_{\mathrm{U}}^{\mathrm{AQCC}}=2.88=, N_{\mathrm{U}}^{\mathrm{QCTB}}=2.37$. AQCC results: Reprinted with permission from Das et al., J. Phys. Chem. A 120, 1625 (2016). Copyright 2016 American Chemical Society. for systems allowing no Kekulé structures was introduced, ${ }^{54}$ and we also make use of this term.

As an example of the system with compensated topological frustration, we consider one of the structures proposed in Ref. 58 for future applications in spintronics. These systems, "Kekulean logic gate structures" (KLGS) in terms of Ref. 58, have triangular zigzag graphene subunits as building blocks. We take the structure given in Fig. 2(b) of Ref. 58 and name it as KLGS $\mathrm{C}_{426}$ (see the structure in our Fig. 8). For the EUE distribution, a usual zigzag-border localization of unpaired electrons is observed. Interestingly, in this instance, the orbital energy gap is very small, but non-vanishing $(0.001$ in $\left|\beta_{0}\right|$ units), whereas the gap equals zero in the individual triangulene building blocks.

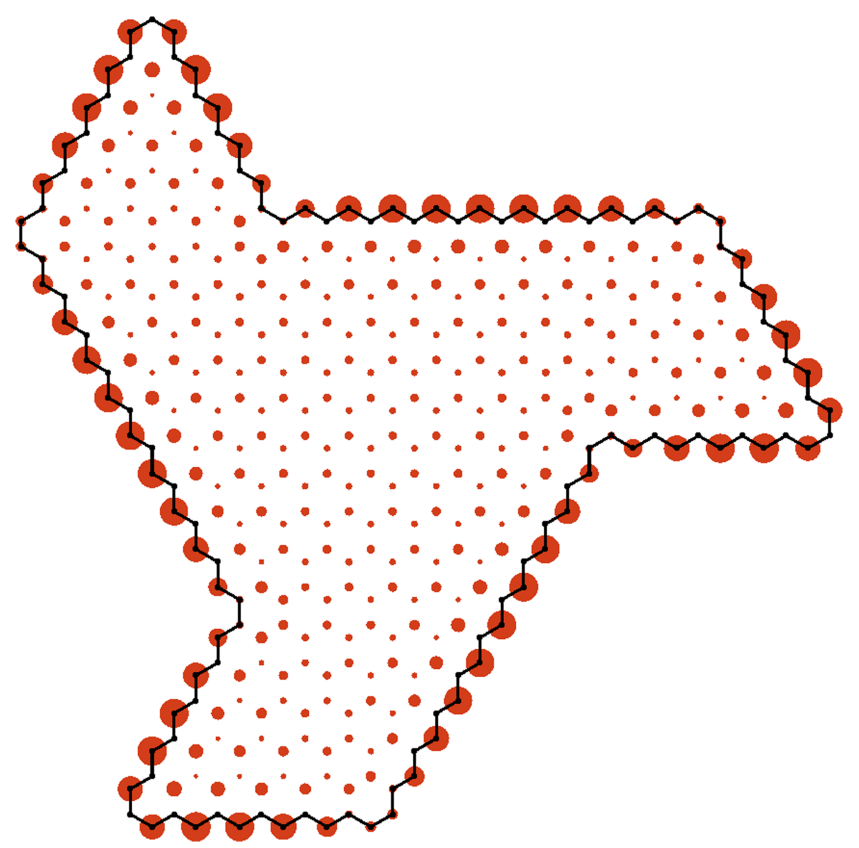

FIG. 8. The QCTB densities of unpaired electron in the triply triangulene structure KLGS C $426 ; N_{\mathrm{U}}^{\mathrm{QCTB}}=18.5$.
Nanoperforated graphene structures represent another sort of systems being actively investigated in the graphene literature (see, e.g., Refs. 35,59-64). We consider a modeled nanographene with pores that somewhat resembles the systems discussed in Ref. 61. This is $(53,16)$ periacene of composition $\mathrm{C}_{8860}$ having 15 hexagonal holes (Fig. 9). In the figure, the corresponding atomic density of unpaired electron is presented. All zigzag-type borders, external and inner, accumulate most of the unpaired electrons. For comparison, the initial $(53,16)$ periacene is shown in Fig. S6. With this, $N_{\mathrm{U}}^{\mathrm{QCTB}}=226.1$, which would be compared with $N_{\mathrm{U}}^{\mathrm{QCTB}}$ $=194.2$ obtained for the nonperforated $(53,16)$ periacene of composition $\mathrm{C}_{10100}$. This result seems to be quite natural since the total length of zigzag borders strongly increases in the perforated structure with the hexagonal holes. Conversely, other EUE measures, the Yamaguchi index $N_{\mathrm{U}}^{\text {Yamaguchi }}$, Eq. (B11), and the Head-Gordon index $N_{\mathrm{U}}^{\mathrm{QCTB}}$ [lin], Eq. (1), come to, seemingly, less adequate values. For instance, in the case of our initial periacene $\mathrm{C}_{10100}$, we find $N_{\mathrm{U}}^{\mathrm{QCTB}}[\operatorname{lin}]=420.2$, and the smaller value $N_{\mathrm{U}}^{\mathrm{QCTB}}[$ lin] $=407.1$ for the perforated structure $\mathrm{C}_{8860}$. In this example, we are faced with the understandable deficiency of Eq. (1) as overestimating

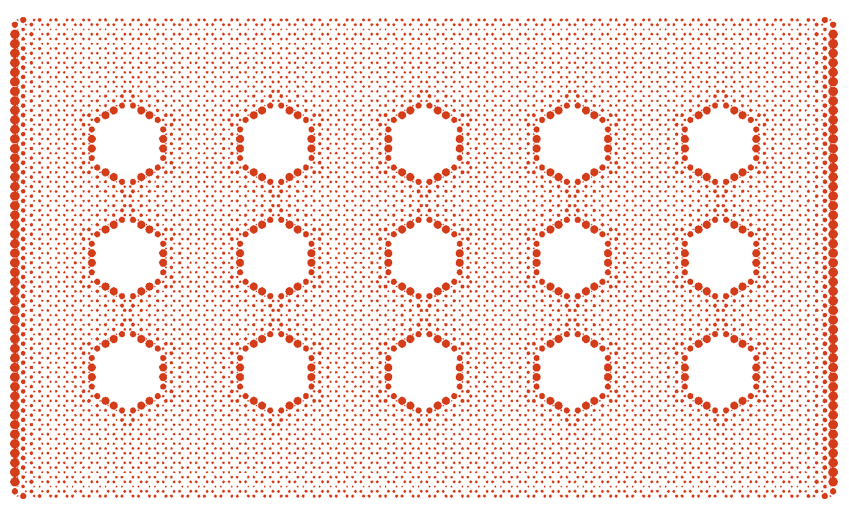

FIG. 9. QCTB densities of unpaired electron in the perforated nanoribbon $\mathrm{C}_{8860}$. 
small dynamical correlation effects, especially for very large networks. It would be interesting to find other examples of such a discrepancy between the linear, Eq. (1), and nonlinear, Eq. (3), EUE indexes. At the same time, specific values $\bar{N}_{\mathrm{U}}^{\mathrm{QCTB}}$ [lin] behave quite reasonably: 0.042 and 0.047 for $\mathrm{C}_{10100}$ and $\mathrm{C}_{8860}$, respectively.

\section{CONCLUDING REMARKS}

In the present paper, we report a significant progress in the improvement of the QCTB algorithm in terms of the efficient treatment of very large molecular systems (10 000 atoms and more) and examined to what extent the simple and computationally highly efficient semiempirical QCTB scheme can reproduce results of the high-level $a b$ initio AQCC theory of unpaired electrons in large conjugated systems. The problem is important because very big systems cannot be easily treated by $a b$ initio bona fide techniques.

Our inference from the comparison we made is the following. By using the QCTB scheme, EUE can be readily understood, at least on a semi-quantitative level. Only the precise details of dynamic correlation, as represented by small NOONs, are obviously not contained in such a simple model. But the most important EUE features are reflected correctly in QCTB. In particular, in both approaches, the ab initio and the semiempirics, NOON spectra of highly correlated large PAHs (e.g., acenes and zigzag graphenes) clearly show crowding occupation numbers near 1 that correspond to fully unpaired electrons and a polyradical nature of the state. At the same time, for weakly correlated systems such as phenacenes and armchair graphenes, there is a significant gap in the NOON spectra (both at the AQCC and QCTB levels) that is in full agreement with the occurrence of a significant $\pi$-electron orbital energy gap in them. Beyond these classes of compounds, comparison with other kinds of systems displaying significantly electron unpairing such as zethrenes and Clar goblet also demonstrates the good performance of QCTB.

We would like to stress that the simplicity of computing EUE properties with QCTB (only Hückel solutions are needed) makes studying gigantic PAHs and polymer structures very easy. Here we improved the QCTB algorithm (Appendixes B and $C$ ) allowing the simulation of ten thousand atom systems even by modest laptops. The only restriction imposed by the method is that the carbon backbone be without odd cycles, that is, be an alternant hydrocarbon. Most systems in the field satisfy this requirement. If not, we must turn to more consistent $\pi$-theories $(\pi$-UHF and $\pi$-CCD) which are much simpler than $a b$ initio models but not so elementary as the QCTB scheme which we scrutinize here. Notice that quite recently there appeared an effective algorithm for reducing the computational work involved in CCD calculations. ${ }^{65}$ But in most cases of very large (nanosized) networks with thousands and more atoms, QCTB seemingly remains the only available and uncomplicated means for producing qualitatively reasonable results.

Closing the concluding remarks, we underline once more that here we compared very different levels of the theory, and all we could expect to obtain is a similarity of trends, rather than exact numerical agreement. In this relation, it is worth mentioning the noteworthy Kutzelnigg's paper ${ }^{66}$ entitled "What I like about Hückel theory." In the paper, it is stated "We come to optimistic conclusions as to the further role of the HMO model, not as an approximation for the solution of the Schrödinger equation, but as a way towards the understanding of some aspects of the Chemical Bond." In a sense, the present paper gives additional arguments in favor of the unaging Hückel discovery deeply rooted in the electron structure theory and chemical topology.

\section{SUPPLEMENTARY MATERIAL}

See supplementary material for analytical expressions for the number of unpaired electrons, comparison of AQCC and QCTB data, figures comparing NOON spectra and densities of unpaired electrons between AQCC and QCTB results, QCTB EUE characteristics, NOON spectra and of densities of unpaired electron.

\section{ACKNOWLEDGMENTS}

This material is based upon work supported by the National Science Foundation under Project No. CHE1213263, by the Austrian Science Fund (SFB F41, ViCom), and by the VSC Research Center funded by the Austrian Federal Ministry of Science, Research and Economy (BMWFW).

\section{APPENDIX A: TWO SIMPLE MODELS OF POLYMER STRUCTURES}

The ability of QCTB to reproduce the principal features of NOON spectra in large-scale PAHs is studied here by using simplistic models of orbital energy spectrum. We will consider two types of the models-gapless and gap-containing systems, and will work with asymptotically large model structures $(N>>1)$. In the case of gapless systems, we assume a discrete uniform distribution for appropriate orbital energies in interval $\left[-\varepsilon_{0}, \varepsilon_{0}\right]$, namely, $\varepsilon_{j}=(2 j / N-1) \varepsilon_{0}$, where $j \leq N$, and $\varepsilon_{0}$ is a certain maximal orbital energy. For $N>>1$, this turns to the continuous uniform distribution $\varepsilon(k)$ (then $j / N \rightarrow k$, and $0 \leq k$ $\leq 1)$, or explicitly,

$$
\varepsilon(k)=(2 k-1) \varepsilon_{0}
$$

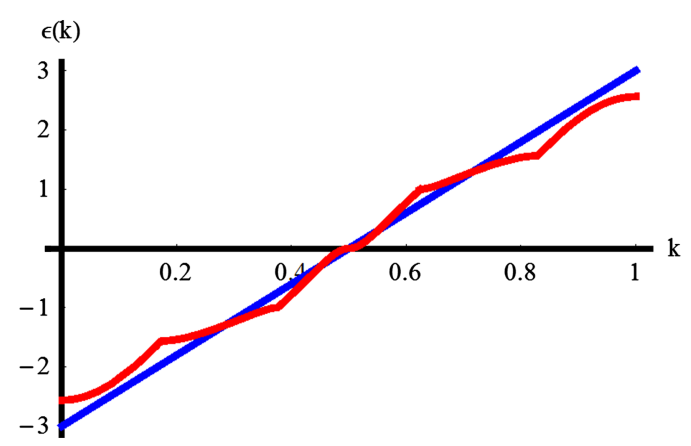

FIG. 10. Plots of the continuous spectrum $\varepsilon(k)$ for model system (blue), Eq. (A1), and quasicontinuous Hückel spectrum for the very large $(n=500)$ polyacene (red). 

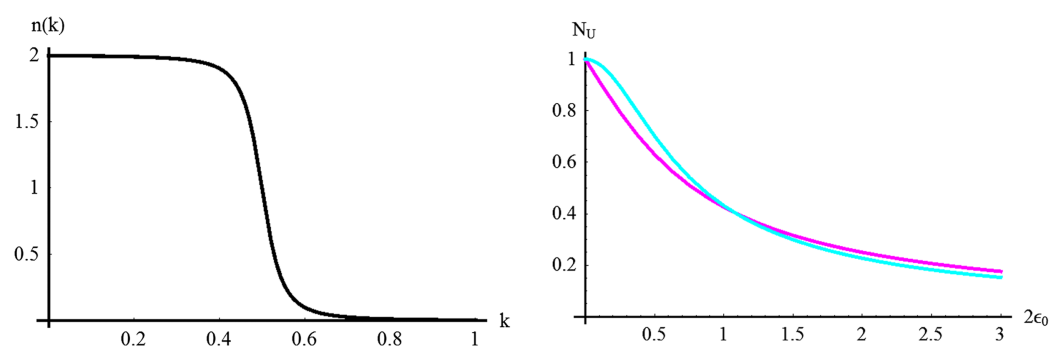

FIG. 11. Left panel: plot of continuous NOON spectrum $n(k)$; right panel: the dependence of $\bar{N}_{\mathrm{U}}^{\mathrm{QCTB}}{ }_{\text {[lin }]}$ (magenta), and $\bar{N}_{\mathrm{U}}^{\mathrm{QCTB}}$ (cyan) on energy width $2 \varepsilon_{0}$.

In specific calculations, we will put the maximal possible $\varepsilon_{0}=3$ (see Section III). As seen from Fig. 10, Eq. (A1) is not a bad approximation of the Hückel energy spectrum for very long polyacenes.

By Eqs. (4) and (5), the corresponding continuous NOON spectrum $n(k)$ is of the form

$$
n(k)=1-(2 k-1) / \sqrt{(2 k-1)]^{2}+\left(\delta / \varepsilon_{0}\right)^{2}} .
$$

Its plot is shown in Fig. 11.

We observe that the $n(k)$ plot is fairly similar to NOON plots for many large PAHs, especially for those of linear acene class and zigzag graphenes ${ }^{12,24,25}$ (see also Fig. S1 of the supplementary material). Besides, we find

$$
\bar{N}_{\mathrm{U}}^{\mathrm{QCTB}}[\operatorname{lin}]=2 \int_{1 / 2}^{1} n(k) d k=1+\delta / \varepsilon_{0}-\sqrt{1+\left(\delta / \varepsilon_{0}\right)^{2}} .
$$

With the adopted parameters $\left(\delta=7 / 24, \varepsilon_{0}=3\right)$ it gives $\bar{N}_{\mathrm{U}}^{\mathrm{QCTB}}[$ lin] $=0.0925$, which is almost the same amount as in Ref. 24 for infinite polyacene $\left(\bar{N}_{\mathrm{U}}^{\mathrm{QCTB}}[\right.$ lin] $=0.098)$. By analogous computations on Eq. (7) we get

$\bar{N}_{\mathrm{U}}^{\mathrm{QCTB}}=\left(\left[1+\left(\varepsilon_{0} / \delta\right)^{2}\right]^{-1}+\left(\delta / \varepsilon_{0}\right) \arctan \left[\varepsilon_{0} / \delta\right]\right) / 2$.

For small $\delta$, we have $\bar{N}_{\mathrm{U}}^{\mathrm{QCTB}}=(\pi / 4) \delta / \varepsilon_{0} \approx \delta$.

With the same adopted values of $\delta$ and $\varepsilon_{0}$ as above it gives 0.0763 that can be compared with our ab initio AQCC value $\bar{N}_{\mathrm{U}}^{\mathrm{AQCC}}=0.087$ for [11] acene. In the right panel of Fig. 11, we show the dependence of both EUE indexes on orbital energy width $2 \varepsilon_{0}$. Both indexes increase significantly with diminishing the width $2 \varepsilon_{0}$, giving practically fully unpaired electrons for a very small width. This formally correct limit case corresponds to unrealistically negligible resonance integrals between all chemically bonded carbon atoms ( $\varepsilon_{0}$ in Eq. (A1) is given, as all $\pi$-orbital energies, in units of resonance integral $\left.\beta_{0}\right)$.

Further, we consider the gap-containing model. In this case, we will obtain the NOON spectrum with its own gap. More specifically, we now take a discontinuous function

$$
\begin{aligned}
\varepsilon(k)= & \varepsilon_{0} / 3 \cdot\{5 k-3, \quad \text { if } 0 \leq k \leq 1 / 2, \\
& \text { or } 5 k-2, \quad \text { if } 1 / 2<k \leq 1\} .
\end{aligned}
$$

In this case, orbital gap $\Delta \varepsilon$ is introduced to be equal to $\Delta \varepsilon=\varepsilon_{0} / 3$ (that is, $\Delta \varepsilon=1$ for $\varepsilon_{0}=3$ ). As we can see in Fig. 12 (the left panel), Eq. (A5) provides a reasonable approximation of the Hückel spectrum in large phenacenes which present a realistic class of the gap-containing PAHs (the phenacenes are discussed in Section IV). In fact, example (A5) is also in line with armchair nanographenes, ${ }^{24,25}$ and large Clar-type PAHs (see Fig. S4 for $\mathrm{C}_{78} \mathrm{H}_{86}$ and Fig. S5 for poly(peritetracene) in the supplementary material). All these features are quite predictable as Eqs. (4) and (5) naturally lead to $n(k)$ near 1 if only the (relative) Hückel energy $\varepsilon(k) \cong 0$.

For computing $\bar{N}_{\mathrm{U}}^{\mathrm{QCTB}}$, we use a suitable relation following from the spectral counterpart of general expression (B1)

$$
\bar{N}_{\mathrm{U}}^{\mathrm{QCTB}}=\delta^{4} \int_{0}^{1}\left(\varepsilon(k)+\delta^{2}\right)^{-2} d k .
$$

Denoting $\Delta=\left(3 \delta / \varepsilon_{0}\right)^{2}$, we get

$\bar{N}_{\mathrm{U}}^{\mathrm{QCTB}}=\frac{\Delta(2 \Delta-3)}{4 \Delta^{2}+5 \Delta+9}+\sqrt{\Delta} \arctan \left[\frac{5 \sqrt{\Delta})}{3+2 \Delta}\right] / 5$.

For typical small values of $\delta$ and the previously defined $\varepsilon_{0}=3$ we have $\bar{N}_{\mathrm{U}}^{\mathrm{QCTB}} \cong 86(\delta / 3)^{4} \approx \delta^{4}$, and this estimation should be compared with $\bar{N}_{\mathrm{U}}^{\mathrm{QCTB}} \approx \delta$ for the gapless system described by Eq. (A4). Thus, the above formal analysis shows that EUE effects in gap-containing systems are much weaker than in the gapless ones. In particular, for the specific value $\delta=7 / 24$ we have from Eq. (A7) $\bar{N}_{\mathrm{U}}^{\mathrm{QCTB}}=0.0054$ [compare this with $\bar{N}_{\mathrm{U}}^{\mathrm{QCTB}}=0.0763$ given by Eq. (A4)].
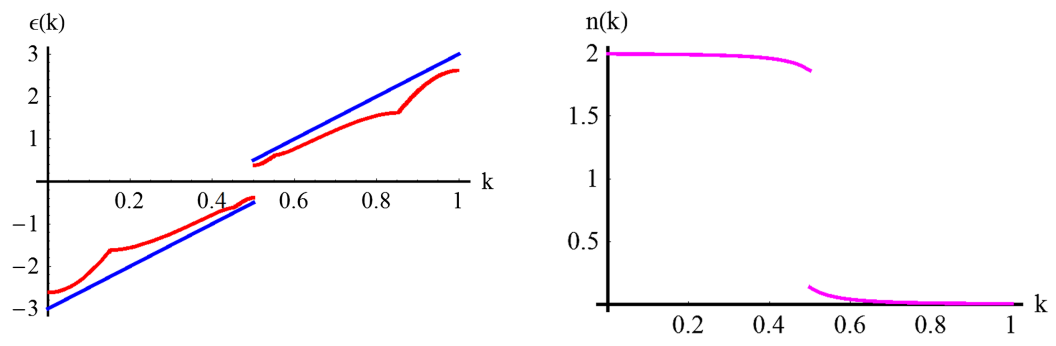

FIG. 12. Left panel: plots of the model discontinuous spectrum $\varepsilon(k)$, Eq. (A5), (blue), and the Hückel spectrum of the very large $(n=500)$ polyphenacene. Right panel: NOON spectrum by Eq. (5) for the model discontinuous $\varepsilon(k)$. 


\section{APPENDIX B: WORKING MATRIX EQUATIONS FOR EUE WITHIN QCTB}

Here we present the new working expressions which are more suitable for quick calculations of the EUE indexes: they avoid the use of spectral formula (7), thus requiring no solution of the Hückel eigenvalue problem. The main result is

$$
N_{\mathrm{U}}=\delta^{4} \operatorname{Tr}\left[\left(h^{2}+\delta^{2} I\right)^{-2}\right],
$$

where we suppress suffix QCTB in most relations given here ( so $N_{\mathrm{U}}=N_{\mathrm{U}}^{\mathrm{QCTB}}$ ). Clearly, only inversion of the known invertible matrix is needed in this formulation. The corresponding EUE density matrix is of the form

$$
D_{\mathrm{U}}=\delta^{4}\left(h^{2}+\delta^{2} I\right)^{-2} .
$$

In above we take $h$ as the conventional Hückel Hamiltonian (in $\left|\beta_{0}\right|$ units), that is, the adjacency (topological) matrix of the associated carbon network. Below we give a short derivation of Eqs. (B1) and (B2), and simultaneously, a concise account of the QCTB model.

We start with specifying the Hückel matrix $h$. Recall that the above relations are only valid for alternant (bipartite) systems which assume the following block structure for the $h$ matrix (see, e.g., Chapter 8 in Ref. 43)

$$
h=-\left(\begin{array}{cc}
0 & B \\
B^{+} & 0
\end{array}\right) \text {. }
$$

In above, the partition into two disjoint sets of atoms ("starred" and "unstarred" graph vertices) is implied, so that every vertex of the first set is only connected to vertices of the second set and vice versa; block $B$ describes all these connections.

The next step is defining Hückel-type Hamiltonians $h^{\alpha}$ and $h^{\beta}$ by which spin-polarization effects of $\alpha$ - (spin-up) and $\beta$ - (spin-down) electrons are introduced in $\mathrm{QCTB}^{24}$

$$
h^{\alpha}=-\left(\begin{array}{cc}
-\delta I & B \\
B^{+} & \delta I
\end{array}\right), h^{\beta}=-\left(\begin{array}{cc}
\delta I & B \\
B^{+} & -\delta I
\end{array}\right) .
$$

Notice that such type of Hamiltonian matrices appeared previously in the Hückel theory of the so-called generalized alternant hydrocarbons. ${ }^{67,68}$ With these Hamiltonians, we can associate density matrices $D^{\alpha}$ and $D^{\beta}$ (projection operators onto occupied $\alpha$ - and $\beta$ - MOs, respectively). For producing such projectors, we make using the elegant Dirac formula ${ }^{69,70}$ For example, $D^{\alpha}=\left(I-h^{\alpha}\left|h^{\alpha}\right|^{-1}\right) / 2$, where $\mid h^{\alpha}=\left[\left(h^{\alpha}\right)^{2}\right]^{1 / 2}$ is an operator modulus of $h^{\alpha}$. $\left|h^{\alpha}\right|$ is easily found by usual work with block matrices. $D^{\beta}$ is evaluated analogously. Together, it leads to the full charge density matrix

$$
D=D^{\alpha}+D^{\beta},
$$

namely,

$$
D=I-h\left(h^{2}+\delta^{2} I\right)^{-1 / 2} .
$$

From this relation, EUE indexes are computed straightforwardly. Additional transformations on this $D$ help us.

Let us introduce the general duodempotency deviation matrix

$$
D_{\text {odd }}=2 D-D^{2},
$$

which determines the EUE density matrix in the Yamaguchi approach. ${ }^{16}$ Then by simple algebra on Eq. (B6), Eq. (B7) simplifies to the transparent result

$$
D_{\text {odd }}=\delta^{2}\left(h^{2}+\delta^{2} I\right)^{-1} .
$$

It remains to take into account that $N_{\mathrm{U}}$ defined by Eq. (3) is the squared norm of $D_{\text {odd }}$ (see Section II)

$$
D_{\mathrm{U}}=\left(D_{\text {odd }}\right)^{2} \text {, }
$$

so

$$
N_{\mathrm{U}}=\operatorname{Tr} D_{\mathrm{U}}=\operatorname{Tr}\left(D_{\text {odd }}\right)^{2} .
$$

This comes to our main results (B1) and (B2) here. For small polyacenes, Eq. (B2) allows compact analytical expressions (see Table $\mathrm{S} 1$ in the supplementary material). We mention in passing that the derivation permits a nonzero spin value in the ground state of the considered alternant molecule. In this case, we have a different number of starred and unstarred atoms, so the topological bond matrix $B$ is rectangular, and this guarantees the occurrence of a certain number of the zero-eigenvalues of the $h$ matrix. ${ }^{43}$ This means that in this case a polyradical structure of the ground state occurs irrespectively of the proper EUE effects.

Returning to Eq. (B7), we recall that in the Yamaguchi approach, ${ }^{16} D_{\text {odd }}$ is the EUE density matrix, and the number of unpaired electrons is simply computed with the help of the formula

$$
N_{\mathrm{U}}^{\text {Yamaguchi }}=\operatorname{Tr}\left(D_{\text {odd }}\right) .
$$

In so doing at the QCTB level, $D_{\text {odd }}$ should be taken from Eq. (B8). Only in case of the linear Head-Gordon index $N_{\mathrm{U}}^{\text {lin }}$, the key relations are cast into a slightly more complicated matrix form. Using Eq. (7) in Ref. 20, it is fairly easy to show that the EUE density matrix $D_{\mathrm{U}}^{\text {lin }}$, by definition having eigenvalues $\lambda_{j}^{\text {lin }}=\operatorname{Min}\left[n_{j}, 2-n_{j}\right] \equiv 1-\left|1-n_{j}\right|$, allows a general matrix representation in terms of $D_{\text {odd }}$ only, namely,

$$
D_{\mathrm{U}}^{\operatorname{lin}}=I-\left(I-D_{\text {odd }}\right)^{1 / 2} \text {. }
$$

Taking into account Eqs. (B12) and (1) in the form

$$
N_{\mathrm{U}}^{\mathrm{lin}}=\operatorname{Tr} D_{\mathrm{U}}^{\operatorname{lin}},
$$

we can also provide a suitable matrix algorithm for the linear version of the Head-Gordon approach. The algorithm is based on using only an iterative construction of the squared matrix root, ${ }^{71}$ but here we omit technical details of such computations.

\section{APPENDIX C: COUNTERPART OF COULSON-RUSHBROOKE PARITY FOR EUE DENSITY MATRIX}

The remarkable Coulson-Rushbrooke parity theorem ${ }^{37}$ can be stated in terms of the charge density matrix as follows: its block matrix form, with the same partition of $\mathrm{AO}$ as in Eq. (B3), is

$$
D=\left(\begin{array}{cc}
I & d \\
d^{+} & I
\end{array}\right)
$$

where $d$ is a matrix of bond orders between starred and unstarred atoms. This result proven in Ref. 37 for the Hückel 
Hamiltonian was extended to more general ones-up to the $\pi$-electron full configuration interaction. ${ }^{72}$ Now we want to clarify which are the consequences of the parity theorem for EUE density matrices. This is easy to do by applying Eq. (C1) to $D_{\text {odd }}$, Eq. (B7). It leads to the representations

$$
D_{\text {odd }}=\left(\begin{array}{cc}
I-d d^{+} & 0 \\
0 & I-d^{+} d
\end{array}\right)
$$

and

$$
D_{\mathrm{U}}=\left(\begin{array}{cc}
\left(I-d d^{+}\right)^{2} & 0 \\
0 & \left(I-d^{+} d\right)^{2}
\end{array}\right) .
$$

The main inference from Eq. (C3) is that EUE atomic densities, which are diagonal elements of $\left(I-d d^{+}\right)^{2}$ and $\left(I-d^{+} d\right)^{2}$, are equally localized on the starred and the unstarred atoms. Indeed, $\operatorname{Tr}\left(I-d d^{+}\right)^{2}=\operatorname{Tr}\left(I-d^{+} d\right)^{2}$ is just a measure of this localization. Incidentally, we mention that according to Ref. 71 , the $\pi$-electron spin-density matrix has a blockdiagonal structure as in Eq. (C3). It serves us as an additional argument in favor of the viewpoint ${ }^{25}$ that for the correlated singlet states, EUE matrix $D_{\mathrm{U}}$ is a counterpart of the spin density matrix (the latter exists for nonzero-spin states only). Interestingly, in case of the restricted open-shell Hartree-Fock (ROHF) method, $D_{\text {odd }}$ defined by Eq. (B7) is exactly converted to the ROHF spin density matrix.

By the above consideration, we are led to even a more efficient computational scheme than given in Appendix B. As the first step, by using appropriate graph-theoretical algorithms, ${ }^{73}$ we divide atoms of the alternant $\pi$-structure into two usual sets of starred and unstarred atoms. Thus, instead of the $h$ matrix we obtain its block $B$ presented in Eq. (B3). At the second step, we compute the two EUE density matrices, $D_{\mathrm{U}}^{*}$ and $D_{\mathrm{U}}^{\circ}$,

$$
D_{\mathrm{U}}^{*}=\delta^{4}\left(B B^{+}+\delta^{2} I\right)^{-2}, D_{\mathrm{U}}^{\circ}=\delta^{4}\left(B^{+} B+\delta^{2} I\right)^{-2} .
$$

The diagonal elements of $D_{\mathrm{U}}^{*}$ and $D_{\mathrm{U}}^{\circ}$ taken together constitute the needed EUE distribution over molecule. At last,

$$
N_{\mathrm{U}}=2 \operatorname{Tr}\left(D_{\mathrm{U}}^{*}\right)^{2} .
$$

Obviously, by this algorithm a size of the matrices used is reduced twofold. As in Appendix B, no matrix diagonalization is needed, if we are interested in $N_{\mathrm{U}}$ and $D_{\mathrm{U}}$ only. If the NOON spectrum is needed, then eigenvalue problem for $B B^{+}$should be solved in addition.

${ }^{1}$ A. Rajca, Chem. Rev. 94, 871 (1994).

${ }^{2}$ A. I. Krylov, Chem. Phys. Lett. 350, 522 (2001).

${ }^{3}$ D. Casanova and M. Head-Gordon, Phys. Chem. Chem. Phys. 11, 9779 (2009).

${ }^{4}$ C. A. Jiménez-Hoyos, T. M. Henderson, T. Tsuchimochi, and G. E. Scuseria, J. Chem. Phys. 136, 164109 (2012).

${ }^{5}$ G. Gidofalvi and D. A. Mazziotti, J. Chem. Phys. 129, 134108 (2008).

${ }^{6}$ K. Pelzer, L. Greenman, G. Gidofalvi, and D. A. Mazziotti, J. Phys. Chem. A 115, 5632 (2011).

${ }^{7}$ J. Hachmann, J. J. Dorando, M. Avilés, and G. K.-L. Chan, J. Chem. Phys. 127, 134309 (2007).

${ }^{8}$ P. G. Szalay, Chem. Phys. 349, 121 (2008).

${ }^{9}$ P. G. Szalay, T. Müller, G. Gidofalvi, H. Lischka, and R. Shepard, Chem. Rev. 112, 108 (2012).

${ }^{10}$ H. Lischka, T. Müller, P. G. Szalay, I. Shavitt, R. M. Pitzer, and R. Shepard, Wiley Interdiscip. Rev.: Comput. Mol. Sci. 1, 191 (2011).

${ }^{11}$ B. Hajgató, D. Szieberth, P. Geerlings, F. De Proft, and M. S. Deleuze, J. Chem. Phys. 131, 224321 (2009).
${ }^{12}$ F. Plasser, H. Pašalić, M. H. Gerzabek, F. Libisch, R. Reiter, J. Burgdörfer, T. Müller, R. Shepard, and H. Lischka, Angew. Chem., Int. Ed. 52, 2581 (2013).

${ }^{13}$ S. Horn, F. Plasser, T. Müller, F. Libisch, J. Burgdörfer, and H. Lischka, Theor. Chem. Acc. 133, 1511 (2014).

${ }^{14}$ A. Das, T. Müller, F. Plasser, and H. Lischka, J. Phys. Chem. A 120, 1625 (2016).

${ }^{15}$ E. L. Wolf, Graphene: A New Paradigm in Condensed Matter and Device Physics (Oxford University Press, 2013).

${ }^{16}$ K. Takatsuka, T. Fueno, and K. Yamaguchi, Theor. Chim. Acta 48, 175 (1978).

${ }^{17}$ D. Doehnert and J. Koutecky, J. Am. Chem. Soc. 102, 1789 (1980).

${ }^{18}$ V. N. Staroverov and E. R. Davidson, Chem. Phys. Lett. 330, 161 (2000).

${ }^{19}$ R. C. Bochicchio, L. Lain, and A. Torre, Chem. Phys. Lett. 374, 567 (2003).

${ }^{20}$ M. Head-Gordon, Chem. Phys. Lett. 372, 508 (2003).

${ }^{21}$ M. Head-Gordon, Chem. Phys. Lett. 380, 488 (2003).

${ }^{22}$ W. Mizukami, Y. Kurashige, and T. Yanai, J. Chem. Theory Comput. 9, 401 (2013).

${ }^{23}$ K. Yoneda, M. Nakano, K. Fukuda, and B. Champagne, J. Phys. Chem. Lett. 3, 3338 (2012).

${ }^{24}$ A. V. Luzanov, in Practical Aspects of Computational Chemistry IV, edited by J. Leszczynski and M. K. Shukla (Springer US, Boston, MA, 2016), pp. 151-206.

${ }^{25}$ A. V. Luzanov, Funct. Mater. 21, 437 (2014).

${ }^{26}$ V. V. Ivanov, I. P. Kisil, and A. V. Luzanov, J. Struct. Chem. 37, 537 (1996).

${ }^{27}$ Z. Cui, H. Lischka, H. Z. Beneberu, and M. Kertesz, J. Am. Chem. Soc. 136, 12958 (2014).

${ }^{28}$ F. B. C. Machado, A. J. A. Aquino, and H. Lischka, Phys. Chem. Chem. Phys. 17, 12778 (2015).

${ }^{29}$ M. Vazdar, M. Eckert-Maksić, and H. Lischka, ChemPhysChem 17, 2013 (2016).

${ }^{30}$ A. V. Luzanov and O. A. Zhikol, Int. J. Quantum Chem. 104, 167 (2005).

${ }^{31}$ A. V. Luzanov and O. V. Prezhdo, J. Chem. Phys. 124, 224109 (2006).

${ }^{32}$ W. Kutzelnigg and V. H. Smith, Int. J. Quantum Chem. 2, 531 (1968).

${ }^{33}$ A. V. Luzanov, J. Struct. Chem. 54, 835 (2013).

${ }^{34}$ W. D. Langer and D. C. Mattis, Phys. Lett. A 36, 139 (1971).

${ }^{35}$ N. N. Tyutyulkov, Int. J. Quantum Chem. 9, 683 (1975).

${ }^{36}$ P.-O. Löwdin, Phys. Rev. 97, 1509 (1955).

${ }^{37}$ C. A. Coulson and G. S. Rushbrooke, Math. Proc. Cambridge Philos. Soc. 36, 193 (1940).

${ }^{38}$ J. Cizek and J. Paldus, J. Chem. Phys. 47, 3976 (1967).

${ }^{39}$ T. Yamada and S. Hirata, J. Chem. Phys. 143, 114112 (2015).

${ }^{40}$ F. Plasser and H. Lischka, J. Chem. Theory Comput. 8, 2777 (2012).

${ }^{41}$ F. Plasser, M. Wormit, and A. Dreuw, J. Chem. Phys. 141, 24106 (2014).

${ }^{42}$ F. Plasser, TheoDORE: A Package for Theoretical Density, Orbital Relaxation, and Exciton Analysis, available from http://theodoreqc.sourceforge.net.

${ }^{43}$ D. M. Cvetkovic, M. Doob, and H. Sachs, in Spectra of Graphs, Theory and Application, 3rd ed., edited by J. Ambrosius Barth (Verlag, HeidelbergLeipzig, 1995).

${ }^{44}$ M. D. Watson, A. Fechtenkötter, and K. Müllen, Chem. Rev. 101, 1267 (2001)

${ }^{45}$ C. A. Coulson, Proc. Phys. Soc. 60, 257 (1948).

${ }^{46}$ M. Baldoni, A. Sgamellotti, and F. Mercuri, Chem. Phys. Lett. 464, 202 (2008).

${ }^{47}$ R. Petersen, T. G. Pedersen, and A.-P. Jauho, ACS Nano 5, 523 (2011).

${ }^{48}$ S. J. Cyvin and I. Gutman, Kekulé Structures in Benzenoid Hydrocarbons (Springer Berlin Heidelberg, Berlin, Heidelberg, 1988).

${ }^{49}$ H. Okamoto, M. Yamaji, S. Gohda, K. Sato, H. Sugino, and K. Satake, Res. Chem. Intermed. 39, 147 (2013).

${ }^{50}$ S. Marković, S. Radenković, Z. Marković, and I. Gutman, Russ. J. Phys. Chem. A 85, 2368 (2011).

${ }^{51}$ P. Hu, S. Lee, T. S. Herng, N. Aratani, T. P. Gonçalves, Q. Qi, X. Shi, H. Yamada, K.-W. Huang, J. Ding, D. Kim, and J. Wu, J. Am. Chem. Soc. 138, 1065 (2016).

${ }^{52}$ P. Yadav, S. Das, H. Phan, T. S. Herng, J. Ding, and J. Wu, Org. Lett. 18, 2886 (2016).

${ }^{53}$ P. Ravat, T. Š olomek, P. Ribar, and M. Juríček, Synlett 27, 1613 (2016).

${ }^{54}$ W. L. Wang, S. Meng, and E. Kaxiras, Nano Lett. 8, 241 (2008).

${ }^{55}$ W. L. Wang, O. V. Yazyev, S. Meng, and E. Kaxiras, Phys. Rev. Lett. 102, 157201 (2009).

${ }^{56}$ Z. Bullard, E. Costa Girão, C. Daniels, B. G. Sumpter, and V. Meunier, Phys. Rev. B 89, 245425 (2014). 
${ }^{57}$ K. Szałowski, J. Appl. Phys. 114, 243908 (2013).

${ }^{58}$ Z. Bullard, E. C. Girão, J. R. Owens, W. A. Shelton, and V. Meunier, Sci. Rep. 5, 7634 (2015).

${ }^{59}$ T. G. Pedersen, C. Flindt, J. Pedersen, N. A. Mortensen, A.-P. Jauho, and K. Pedersen, Phys. Rev. Lett. 100, 136804 (2008).

${ }^{60}$ T. G. Pedersen and J. G. Pedersen, J. Appl. Phys. 112, 113715 (2012).

${ }^{61}$ J. G. Pedersen, T. Gunst, T. Markussen, and T. G. Pedersen, Phys. Rev. B 86, 245410 (2012).

${ }^{62}$ M. Kim, N. S. Safron, E. Han, M. S. Arnold, and P. Gopalan, Nano Lett. 10, 1125 (2010).

${ }^{63}$ L. P. Biró, P. Nemes-Incze, and P. Lambin, Nanoscale 4, 1824 (2012).

${ }^{64}$ P. Vancsó, G. I. Márk, P. Lambin, A. Mayer, C. Hwang, and L. P. Biró, Appl. Surf. Sci. 291, 58 (2014).
${ }^{65}$ J. Brabec, C. Yang, E. Epifanovsky, A. I. Krylov, and E. Ng, J. Comput. Chem. 37, 1059 (2016).

${ }^{66}$ W. Kutzelnigg, J. Comput. Chem. 28, 25 (2007).

${ }^{67}$ T. K. Rebane, Vestnik Leningradskogo Universiteta, Ser. Fizika i Khimia 22, 30 (1962).

${ }^{68}$ M. M. Mestechkin, Theor. Exp. Chem. 1, 388 (1967).

${ }^{69}$ P. A. M. Dirac, Math. Proc. Cambridge Philos. Soc. 30, 150 (1934).

${ }^{70}$ A. S. Davydov, Quantum Mechanics 1st American ed. (Pergamon Press, 1965).

${ }^{71} \mathrm{~J}$. Higham, Functions of Matrices: Theory and Computation (SIAM, Philadelphia, PA, 2008).

${ }^{72}$ A. D. McLachlan, Mol. Phys. 2, 271 (1959).

${ }^{73}$ Graph Theory and Computing, edited by R. Read (Academic Press, New York, 1972). 